\title{
DETERMINATION OF MOST FREQUENT EMERGENCIES IN PATIENTS WITH FIXED AND REMOVABLE ORTHODONTIC APPLIANCES AT TERTIARY CARE DENTAL HOSPITAL
}

\author{
Syed Salman Shah ${ }^{1}$, Kawish Syed ${ }^{2}$, Zafar ul Islam ${ }^{3}$, Shahab Adil ${ }^{4}$
}

\section{$\underline{A B S T R A C T:}$}

\section{OBJECTIVES:}

To determine the frequency of emergencies in patients with fixed and removable orthodontic appliances at tertiary care dental hospital.

\section{METHODOLOGY:}

A questionnaire was designed for this descriptive cross-sectional study to be filled by the clinician (L3/L4 FCPS resident) at the end of addressing every orthodontic emergency. Sampling was done under consecutive nonprobability protocols. Descriptive statistics were applied to determine the frequency of different orthodontic emergencies, and Pearson's chi-square test was applied to determine association of emergencies with gender and etiology of emergency (patient related vs operator related). Data was analyzed on SPSS version 20.

\section{RESULTS:}

A total of 175 patients reported with orthodontic emergencies. The sample comprised $38.3 \%$ males and $61.7 \%$ females. Most frequent orthodontic emergency reported was deboned brackets in fixed appliances, while the most common emergency in removable appliances was traumatic PNAM. A statistically significant association (Pearson's Chi Square $=4.74$, Cramer's V=0.165, $p=0.029$ ) was seen for removable and fixed appliance emergencies with males and females.

\section{CONCLUSION:}

Most frequent fixed appliance orthodontic emergencies were deboned brackets while for removable appliance emergencies were trauma due to PNAM. Emergencies with removable appliances were mostly due to the operator related factors, while in fixed appliances patient related factors were dominating.

KEYWORDS: Orthodontic Emergencies, Fixed Appliances, Removable Appliances

How to cite this article:

Shah SS,Syed K, Islam ZU, Adil S. Determination of Most Frequent Emergencies in Patients with Fixed and Removable Orthodontic Appliances at Tertiary Care Dental Hospitals. J Gandhara Med Dent Sci. 2022;9(1): 69-74

https://doi.org/10.37762/jgmds.9-1.267

\section{Correspondence:}

${ }^{4}$ Shahab Adil, Professor and Head Department of Orthodontics, PDC, Peshawar

(v): $+92-343-9009494$

$凶: \quad$ shahabadil@hotmail.com

${ }^{1}$ Post Graduate FCPS-II Trainee, Department of Orthodontics, PDC, Peshawar.

${ }^{2}$ Assistant Professor, Department of Periodontology, SBDC,

Peshawar

${ }^{3}$ Associate Professor, Department of Orthodontics, PDC, Peshawar

\section{INTRODUCTION:}

Emergency, as defined by the American College of Emergency Physicians (ACEP), is any condition perceived by the prudent layperson, or someone on his or her behalf, as requiring immediate medical or surgical evaluation and treatment ${ }^{1}$. Orthodontic emergencies cannot be regarded as real emergencies but are encountered often by an orthodontists or dentist in first few 
weeks of treatment or even afterwards. The most frequent emergencies patient reports are tooth pain, bracket breakage, loose bands, wire poking, tooth mobility, ulcers or soreness in the mouth, bleeding gums, missing elastics or ties, broken/lost appliance and a piece of an appliance getting swallowed $^{2}$. A wide range of contemporary orthodontic appliances (removable/fixed) is used to achieve orthodontic objectives. Expert manipulation of appliances will not only help in achieving objectives efficiently but also decrease patient discomfort, and apprehensions, soft/hard tissue damage, treatment duration and $\operatorname{cost}^{2-6}$. Causes of the emergencies are numerous, but can be broadly divided into operators and patient negligence ${ }^{7}$. Literature suggests the most frequent orthodontic emergency is debonded brackets while broken removable appliances require repair or remake, ${ }^{8}$ being second most frequent, followed by Arch wire problems ${ }^{4,5,7,9}$. Prevalence of debonded brackets was reported highest in patients treated with fixed appliances of age 14-16 years at lower buccal segment including both the sexes ${ }^{9}$. While some studies indicate bracket breakage to be more common in maxillary $(36.5 \%)$ buccal region than in mandible $(22.8 \%)$, the lower anterior segment had the lowest percentage of bracket breakage ${ }^{10,11}$. As orthodontic emergencies can be multifactorial, a study of orthodontic patients at tertiary care hospital of Peshawar is warranted, due to the different food habits and musculoskeletal patterns of the population as well as difference in protocols followed by different hospitals. The aim of this study is to determine the frequency of emergencies in patients with fixed and removable orthodontic appliances at tertiary care dental hospital of Peshawar.

\section{METHODOLOGY:}

This prospective cross-sectional study was carried out at the Department of Orthodontics, Peshawar Dental College, Peshawar, after approval from the Institutional Review Board. A Performa was designed for this descriptive cross-sectional study in order to be filled by the clinician at the end of addressing every orthodontic emergency. Only those patients who reported to the department without a formal appointment with complaints paint, trauma or any other complaint regarding appliances and were treated by R3/R4 level residents were included in this study. All those patients who were under treatment from other hospitals or clinics, patients who didnet know how this happened and those who reported on unscheduled appointments without any complaints were excluded. Sample size was 175 using consecutive sampling techniques. Study duration was 12 months from 5th December 2018 to 4th December 2019. Cause of the emergency was considered as due to operator related factors if the cause of the emergency was due to the following: I could feel something between my teeth after last visit, it occurred before eating any food, wire was poking my cheek, appliance edges were sharp. While it was due to patient related factors, if it occurred due to one of the following reasons: my appliance fell from the high place and broke, my maid threw the appliance in the dustbin, I took a big bite, ate hard food, I took a bite with my front teeth. Descriptive statistics were applied to determine the frequency of different orthodontic emergencies, and Pearson "es chi-square test was applied to determine association of emergencies with gender and etiology of emergency (patient related vs operator related).

\section{RESULTS:}

Sample of 175 patients comprised of $38.3 \%$ (67) males and $61.7 \%$ (108) females. Figure 1 shows the frequencies of various emergencies that were reported. Fifteen $(8.6 \%)$ subjects presented with emergencies related to removable appliances while $160(91.4 \%)$ subjects presented with fixed appliance therapy emergencies. The most frequent emergency reported was debonded brackets $60.6 \%$ (106). The other emergencies reported were decemented molar bands $20.0 \%$ (35), traumatic wire/auxiliary $8.6 \%$ (15), traumatic PNAM, dislodged ties/separators $7.4 \%$ (13), traumatic jiggered power chain $2.9 \%$ (5), traumatic removable appliance $0.6 \%$ (1). Table 1 shows the relationship of various removable and fixed appliances emergencies with etiology and the genders of the subjects. A statistically significant association (Pearson ${ }^{\text {ee }}$ s Chi Square $=4.74$, Cramer ${ }^{\text {ee }} \mathrm{S}$ $\mathrm{V}=0.165, \mathrm{p}=0.029$ ) was seen for removable and fixed appliance emergencies with males and females. The presentation of both removable and fixed appliance emergencies is more frequent in females than male subjects. The fixed appliances emergencies were more frequent due to patients related factors while the operator related factors were more frequent in removable appliances emergencies especially trauma from NAM appliances. 


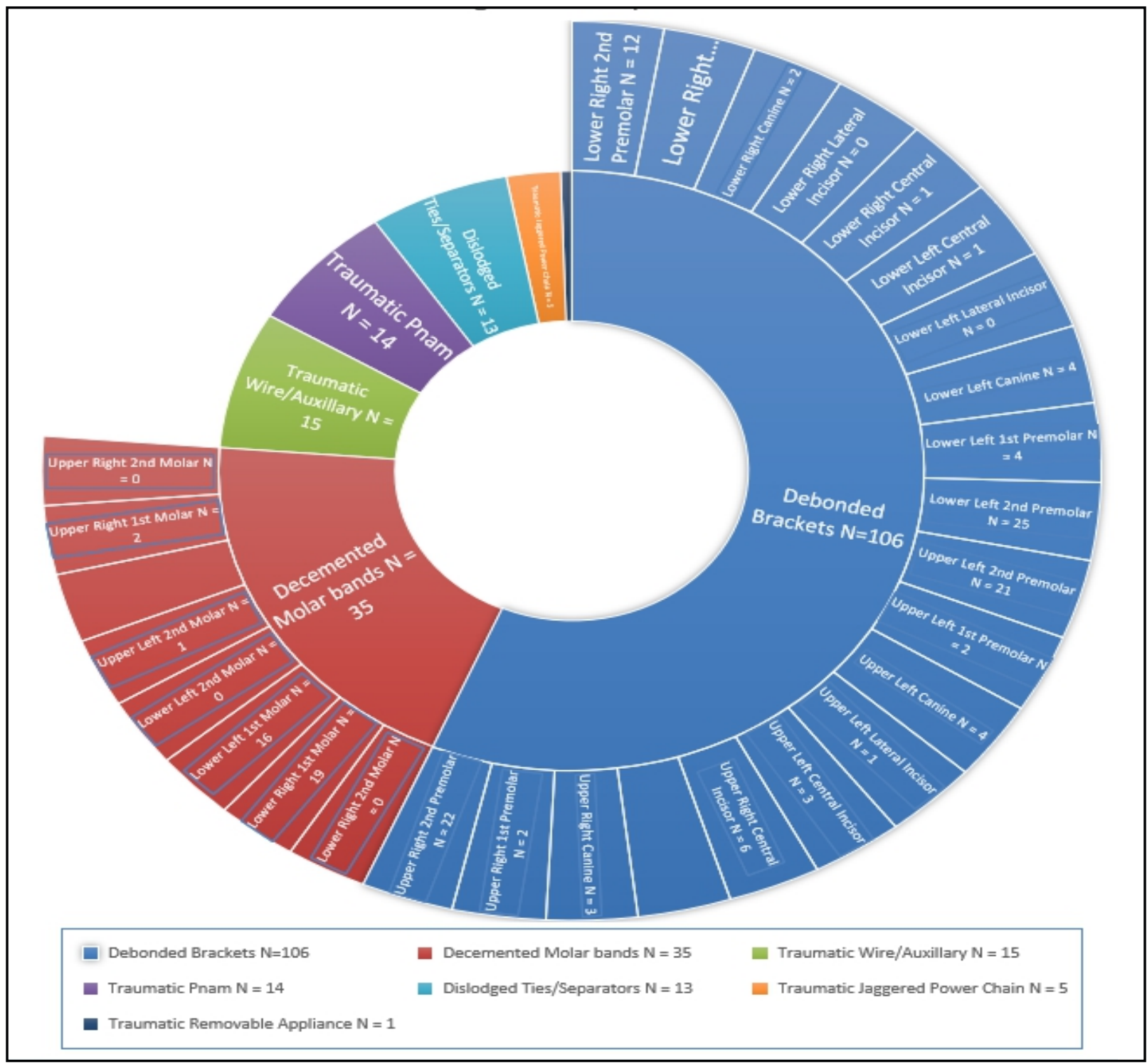

Figure 1: Frequency of Different Emergencies Associated with Removable and Fixed Appliances

Table 1: The Relation of Removable and Fixed Appliance Emergencies with Etiology and Gender of Subjects (N=175)

\begin{tabular}{|l|l|l|l|l|}
\hline Gender & & $\begin{array}{l}\text { Patient Related } \\
\text { Factors }\end{array}$ & $\begin{array}{l}\text { Operator } \\
\text { Related Factors }\end{array}$ & Total \\
\hline Male & Observed & 48 & 19 & 67 \\
& Expected & 53.6 & 13.4 & 67 \\
\hline \multirow{2}{*}{ Female } & Observed & 92 & 16 & 108 \\
\hline \multirow{2}{*}{ Total } & Expected & 86.4 & 21.6 & 108 \\
\hline
\end{tabular}

Pearson's Chi Square $=4.74, \mathrm{df}=1, \mathrm{p}=0.029$

Cramer's V $=0.165, \mathrm{p}=0.029$ 


\section{DISCUSSION:}

Orthodontic emergencies are frequent in patients receiving therapy for malocclusion either with removable or fixed appliances. The emergencies in orthodontics are not life-threatening conditions however; they cause considerable discomfort to the patients. The orthodontic emergencies need immediate attention to relieve the patientes discomfort but the coverage of the orthodontic care in terms of timings is limited in the evening and at night. Therefore, the categorization of the most frequent orthodontic emergencies is necessary to determine the cause and solutions to discomforting problems and enhancing the quality of care. The present study was carried out to determine the most frequent orthodontic emergencies and their causes. The present study shows that the most frequent emergency was breakage of brackets in patients with fixed appliances. This finding has been consistent with other studies, ${ }^{12-14}$ carried out for determination of most frequent orthodontic emergencies. The bonding failure was most common in adults in the present study, which is in contrast to other studies, ${ }^{12,13}$ that has shown more bonding failures in adolescence. This difference may be due to the variation in the lifestyle of the subjects, especially the eating habits ${ }^{12}$. Assessing de cemented molar bands and debonded brackets individually; it was found that most common debonded brackets emergencies occurred on the lower left quadrants with most common teeth being second premolars. The incidence of de cemented molar bands was highest in the lower right quadrants in the first molars. Similar results were recorded in previous studies, ${ }^{15,16}$ whereas contradicting results were noticed in a study, ${ }^{17}$ on frequency of bracket breakage and bond failure in patients undergoing fixed orthodontic treatment. This finding of higher incidence of debonded brackets on the left side may well be attributed to the study, ${ }^{18}$ indicating that subjects with a dominant right hand may efficiently maintain oral hygiene on the opposite side and inadvertently causing bracket breakage. Our study indicates that all the subjects were right-handed. Therefore, it permits us to maintain that force application on teeth while brushing may inadvertently debonded brackets on the left quadrants due to a dominant right hand. The decemented band may be attributed to use of elastics, poor band fit, poor isolation and banding techniques of the operator. Orthodontic removable and fixed appliances require careful handling at chair side by operator followed by strict instruction compliance by the patients. Any noncompliance on behalf of the operator or patients may lead to orthodontic emergencies. In the present study the most common orthodontic emergency due to operator related factors was removable appliance (PNAM), while bracket breakage was the most frequent emergency due to patient noncompliance with post-operative instructions. Similar results have been shown by other studies, ${ }^{12,16}$ in which the bracket failure in fixed appliances was most frequent. However, the cause of bracket bond failure has been differently mentioned. Some studies, ${ }^{18}$ showed the operators related factors like poor bonding techniques while the others have found the patients noncompliance with post bonding instructions to be the main cause of bracket bond failures ${ }^{12}$. While considering the most frequent orthodontic emergencies, the less frequent should not be overlooked, as above all, they present as an emergency and cause considerable discomfort to the patients. The cause of these less frequent emergencies also needed to be properly evaluated for operator and patient related factors. In this present study most of the less frequent emergencies like trauma jiggered end of power chain; traumatic wires, traumatic PNAM and dislodged molar bands were due to operator related factors. Therefore, to minimize orthodontic emergencies, the clinicians are required to strictly exercise the procedural guidelines of fixed and removable appliances.

\section{LIMITATIONS:}

The answers of the participants may have been influenced by Hawthrone effect resulting in alteration in their behavior due to their awareness of being appraised ${ }^{19}$. There was no attempt to standardize the duration of treatment time, as the patient needed a minimum of 3-6 months to adapt to their appliance. This may have led to inconsistency in the answers. It was a single centered study. More controlled studies are required to generate authentic results.

\section{CONCLUSION:}

Most frequent fixed appliance orthodontic emergencies were deboned brackets while for removable appliance emergencies were trauma due to PNAM. Emergencies with removable appliances were mostly due to the operator related factors, while in fixed appliances patient related factors were dominating

CONFLICT OF INTEREST: None 
FUNDING SOURCES: None

\section{REFERENCES:}

1. Shayamala N, Anand S. Management of orthodontic emergencies-to act or not?. Int $\mathrm{J}$ Oral Health Dent. 2020;4(3):205-7.

3. Gyawali R, Pokharel PR, Giri J. Emergency appointments in orthodontics. APOS Trends Orthod. 2019;9(1):40-3.

4. Makhbul MZ, Hassan WN. A clinical audit of the success rate of removable functional appliances treatment. Malays Dent J. 2019;1:61-73.

5. Gbolahan OO, Ogunmuyiwa SA, Olowookere SA, Ayantunde AA, Ogundipe KO. Factors associated with non-attendance at early review appointments after cleft lip and palate repair in a Nigerian specialist maxillofacial centre. East Cent Afr J Surg. 2019;24(1):40-6.

6. Proffit WR, Fields HW, Larson BE, Sarver DM. Contemporary Orthodontics. 6th ed. Philadelphia: Elsevier; 2018.

7. Caprioglio A, Pizzetti GB, Zecca PA, Fastuca R, Giuliano M, Nanda R. Management of orthodontic emergencies during 2019-NCOV. Prog Orthod. 2020;21(10):1-4.

8. Dowsing P, Murray A, Sandler J. Emergencies in orthodontics-part 2: management of removable appliances, functional appliances and other adjuncts to orthodontic treatment. Dent Update. 2015;42(3):221-8.

9. Stasinopoulos D, Papageorgiou SN, Kirsch F, Daratsianos N, Jäger A, Bourauel C. Failure patterns of different bracket systems and their influence on treatment duration: a retrospective cohort study. Angle Orthod. 2018;88(3):338-47.

10. Zegan G, Golovcencu L, Anistoroaei D. Undergraduate orthodontic education and dental students ${ }^{\text {ee }}$ performances-a survey. Int $\mathrm{J}$ Med
12. Barbosa IV, Ladewig VM, Almeida Pedrin RR, Cardoso MA, Santiago Junior JF, Conti AC. The association between patient's compliance and age with the bonding failure of orthodontic brackets: a cross-sectional study. Prog Orthod. 2018;19(1):11.

Dent. 2019;23(2):237-43.

Kafle D, Mishra RK, Hasan MR, Saito T. A retrospective clinical audit of bracket failure among patients undergoing orthodontic therapy. Int $\mathrm{J}$ Dent. 2020;2020:1-5.

Almosa $\mathrm{N}$, Zafar $\mathrm{H}$. Incidence of orthodontic brackets detachment during orthodontic treatment: a systematic review. Pak J Med Sci. 2018;34(3):744.

14. Al-Groosh D, Al-Khatieeb MM, Akrem LA. The perception of fixed orthodontic treatment and components amongst a sample of specialist orthodontists in Baghdad city. Int $\mathrm{J}$ Sci Res. 2017;6(9):543-7.

15. Shaeran TA, Samsudin AR. Dislodged bonded molar tube into wound during orthognathic surgery. Case Rep Dent. 2018;2018:1-4.

16. Asefi S, Aslani M, Kamali E, Bahrami R, Mirhashemi SA. Comparing shear bond strength and adhesive remnant index between light cure composite, no mix composite, and no bond composite; an in vitro study. J Mazandaran Univ Med Sci. 2021;31(202):116-25.

17. Agbaje HO, Kolawole KA, Otuyemi OD. Evaluation of early changes in oral health-related quality of life amongst Nigerian patients undergoing fixed orthodontic appliance therapy. Int Orthod. 2018;16(3):571-85.

18. Hasan A. Frequency of bracket bond failure in orthodontic patients with normal over bite and deep bite. J Bahria Univ Med Dent Coll. 2017;7(2):82-5.

19. Abreu LG, Santos TR, Melgaço CA, Abreu MH, Lages EM, Paiva SM. Impact of orthodontic treatment on adolescentse $^{\text {ee }}$ quality of life: a longitudinal evaluation of treated and untreated individuals. Qual Life Res. 2018;27(8):2019-26. 


\section{CONTRIBUTORS}

1. Syed Salman Shah - Data Acquisition; Drafting Manuscript; Critical Revision

2. Kawish Syed - Data Analysis/Interpretation; Drafting Manuscript; Critical Revision

3. Zafar ul Islam - Data Analysis/Interpretation; Drafting Manuscript

4. Shahab Adil - Concept \& Design; Critical Revision; Supervision; Final Approval

LICENSE: JGMDS publishes its articles under a Creative Commons Attribution Non-Commercial Share-Alike license (CC-BY-NC-SA 4.0). COPYRIGHTS: Authors retain the rights without any restrictions to freely download, print, share and disseminate the article for any lawful purpose. It includes scholarly networks such as Research Gate, Google Scholar, LinkedIn, Academia.edu, Twitter, and other academic or professional networking sites. 